\title{
A study of semi-insulating GaN grown on AlN buffer/sapphire substrate by metalorganic chemical vapor deposition
}

\author{
Hongbo Yu ${ }^{\mathrm{a}, *}$, M. Kemal Ozturk ${ }^{\mathrm{b}}$, Suleyman Ozcelik ${ }^{\mathrm{b}}$, Ekmel Ozbay ${ }^{\mathrm{a}, \mathrm{c}, \mathrm{d}}$ \\ ${ }^{a}$ Nanotechnology Research Center, Bilkent University, Bilkent, 06800 Ankara, Turkey \\ ${ }^{\mathrm{b}}$ Department of Physics, Gazi University, Bahcelievler, Ankara 06500, Turkey \\ ${ }^{\mathrm{c}}$ Department of Physics, Bilkent University, Bilkent, 06800 Ankara, Turkey \\ ${ }^{\mathrm{d}}$ Department of Electrical and Electronics Engineering, Bilkent University, Bilkent, 06800 Ankara, Turkey
}

Received 11 February 2006; received in revised form 18 April 2006; accepted 13 May 2006

Communicated by K.M. Lau

Available online 14 July 2006

\begin{abstract}
We report the remarkably improved crystal quality of semi-insulating GaN grown by metalorganic chemical vapor deposition on an AlN buffer layer, which is deposited on sapphire substrate. The electrical and structural properties are characterized by dark current-voltage transmission line model and X-ray diffraction measurements. It is found that the crystal quality of the GaN epilayer is strongly related with the growth temperature of the decreased-temperature GaN interlayer. In comparison with the normal GaN grown on sapphire, the crystal quality is remarkably improved along with a semi-insulating electrical character. The high-mobility field effect transistors device based on the semi-insulating GaN shows good pinch off properties. Our electrical measurement results of GaN grown directly on an AlN buffer indicated that the as-grown-undoped GaN is naturally semi-insulating material. The origination of the residual donors in normal GaN grown on sapphire substrate is also discussed.
\end{abstract}

(C) 2006 Elsevier B.V. All rights reserved.

PACS: 81.15.Gh; 71.55.Eq; 72.20.-i

Keywords: A1. Dislocation; A1. X-ray diffraction; A3. AlN buffer; A3. MOCVD; B1. III-V nitrides

\section{Introduction}

III-Nitride based high mobility field effect transistors (HEMT) are attracting great interest due to their capability of operating at high power, temperature, and frequency [1,2]. The epitaxy structure of HEMT is in principle rather simple, which consists of a thin AlGaN layer on top of a GaN baselayer. To enable a complete channel pinch-off, low loss at high frequencies, and proper drain-source current saturation, the achievement of the semi-insulating $\mathrm{GaN}$ (SI-GaN) template is critically important for this kind of device [3]. It is well known that normally undoped GaN grown on sapphire by metalorganic chemical vapor deposition (MOCVD) typically exhibits n-type conductivity. The origin of the residual donors is not

\footnotetext{
*Corresponding author. Tel.: + 90312 2901020; fax: + 903122901015.

E-mail address: yu@fen.bilkent.edu.tr (Hongbo Yu).
}

altogether clear at present, presumably due to oxygen impurity diffusion from the sapphire substrate or unintentionally introduced during growth $[4,5]$. In order to reliably obtain SI-GaN grown on sapphire substrate it is rather significant to clarify the cause of the residual donors.

Due to the residual donors, SI-GaN is typically grown by compensating the donors with acceptor states, which is introduced by intentional doping with $\mathrm{C}, \mathrm{Fe}$ and $\mathrm{Cr}$, or high density of edge-type dislocations [6-10]. However, $\mathrm{Fe}$ and other heavy metals tend to have reactor contamination issues leading to rather strong memory effects [7]. Moreover, high dislocation densities are undesirable in the GaN epitaxy structure, either because of the deep trapping effects due to current collapse while applying high drain voltage, or because of negative effects on surface morphography and consequently the interface roughness of the heterostructure $[11,12]$. Therefore, the performance of the 
HEMT device is expected to be significantly improved by reducing defect densities in SI-GaN layer.

Recently, the success in the growth of high-temperature AlN film on sapphire provides a more encouraging solution for achieving SI-GaN layers [13]. In the present study, we demonstrated the highly improved crystal quality of SI-GaN by way of the insertion of a decreased temperature $\mathrm{GaN}$ interlayer between an HT-GaN and AlN buffer. The origination of the residual donors in normal GaN grown on sapphire is also discussed.

\section{Experiments}

The samples in this study were all grown on double polished $c$-plane sapphire by a low-pressure MOCVD system. Hydrogen was used as the carrier gas, and Trimethylgallium (TMGa), trimethylaluminum (TMAl), and ammonia were used as $\mathrm{Ga}, \mathrm{Al}$ and $\mathrm{N}$ precursors, respectively. The GaN layers were grown on either hightemperature AlN buffer, or low-temperature GaN nucleation layer. A $15 \mathrm{~nm}$-thick AlN nucleation layer was deposited at $840{ }^{\circ} \mathrm{C}$, and then the reactor temperature was ramped to $1150{ }^{\circ} \mathrm{C}$ and the AlN buffer layer was grown, followed by 2-min growth interruption in order to reach growth conditions for $\mathrm{GaN}$. The growth parameters of high-temperature GaN (HT-GaN) were as follows: growth temperature $1080^{\circ} \mathrm{C}$, reactor pressure $200 \mathrm{mbar}$, and growth rate about $2 \mu \mathrm{m} \mathrm{h}^{-1}$. In some of the samples, a $0.5 \mu \mathrm{m}$ thick decreased temperature $\left(1000-1050{ }^{\circ} \mathrm{C}\right) \mathrm{GaN}$ (named herein DT-GaN) layer was grown between AlN buffer and HT-GaN. A normal undoped GaN sample was prepared on sapphire substrate with a $25 \mathrm{~nm}$-thick GaN nucleation layer deposited at $500{ }^{\circ} \mathrm{C}$. For a fair comparison, the thickness and growth parameters of the HT-GaN were kept constant for all of the samples.

The structural properties of the samples were characterized by the high-resolution X-ray diffraction (XRD) technique using a Bruker D8 system, delivering a $\mathrm{CuK}_{\alpha} 1$ line. A slit of $0.5 \mathrm{~mm}$ was put in front of the detector in order to rule out the broadening effect due to the limited sub-grain size and inhomogeneous strain in double-axis rocking scans. To obtain the resistivity of SI-GaN, the dark current-voltage $(I-V)$ transmission line model (TLM) measurement was performed. The TLM patterns (prepared by the deposition of $\mathrm{Ti} / \mathrm{Al} / \mathrm{Ni} / \mathrm{Au}(100 / 400 / 100 / 400 \AA)$ contact) used for sheet resistivity calculations consisted of a set of $200 \times 100 \mu \mathrm{m}$ pads with space in the 5-50 $\mu \mathrm{m}$ range. A thin $(25 \mathrm{~nm}) \mathrm{Al}_{0.3} \mathrm{Ga}_{0.7} \mathrm{~N}$ layer was grown on the $\mathrm{SI}-\mathrm{GaN}$ samples. The presence of two-dimensional electron gas at the $\mathrm{AlGaN} / \mathrm{GaN}$ interface insures an ohmic contact, as proposed in Ref. [7]. After this, the AlGaN layer between the contacts was etched off with reactive ion etching. Dark $I-V$ characterization of contact pads were carried out by an HP 4142B semiconductor parameter analyzer. To explore the concentration of the impurities in the samples, secondary ion mass spectroscopy (SIMS) analysis of the selective samples were done in the Evans Analytical Group.

\section{Results and discussions}

Fig. 1 shows the $I-V$ characteristics of $\mathrm{GaN}$ grown on AlN buffer obtained from a set of TLM pads. After rapid thermal annealing at $750{ }^{\circ} \mathrm{C}$ for $1 \mathrm{~min}$, the linearity of $I-V$ curves were obtained for all the TLM pads. At a bias of $10 \mathrm{~V}$, the dark remains about $2 \times 10^{9} \mathrm{~A}$ between two TLM pads separated by a $5 \mu \mathrm{m}$ gap. The $I-V$ characteristics demonstrate that GaN layer grown on AlN buffer/sapphire substrate exhibit a semi-insulating character with calculated sheet resistivity of $3.26 \times 10^{11} \Omega \mathrm{sq}^{-1}$. However, the Hall measurement of normal undoped GaN layer shows an n-type background with sheet resistivity of $8.7 \times 10^{3} \Omega \mathrm{sq}^{-1}$ (electron concentration about $2 \times 10^{16} \mathrm{~cm}^{-3}$ ), which is consistent with the previous reports $[7,14]$. Because the growth parameters of the HT-GaN were kept constant for the two samples, the electrical characters of the two samples reveal that residual donors in $\mathrm{GaN}$ grown on sapphire is not due to impurities atoms (such as $\mathrm{O}$ and $\mathrm{Si}$ ) incorporation during high-temperature growth.

Fig. 2(a) and (b) are the SIMS profiles of impurities $(\mathrm{O}$ and $\mathrm{C})$ recorded from cross-sectional normal and SI-GaN samples, respectively. As shown in Fig. 2(a), the oxygen concentration is very high at the interface of LTGaN and the sapphire substrate $\left(>10^{20} \mathrm{~cm}^{-3}\right)$. From the interface to approx. $0.2 \mu \mathrm{m}$ away from the sapphire substrate, the oxygen concentration is decreased gradually to about $2-5 \times 10^{16} \mathrm{~cm}^{-3}$, indicate that the substrate itself is the oxygen source. During the initial growth stage, oxygen can move into the GaN layer by thermal etching and/or diffusion, which results in a highly conducting channel near the sapphire interface $[14,15]$. Away from this region to the surface, the oxygen concentration is kept relatively stable at approx. $5 \times 10^{16}-1 \times 10^{17} \mathrm{~cm}^{-3}$. As shown in Fig. 2(b), the oxygen concentration in the III-nitride epitaxy layer shows strong dependence on the III-elements. In the AlN buffer, the oxygen concentration is relatively high $\left(5 \times 10^{18}-1 \times 10^{20} \mathrm{~cm}^{-3}\right)$ due to high reactivity between $\mathrm{Al}$ and $\mathrm{O}$. While in the $\mathrm{GaN}$ layer, the

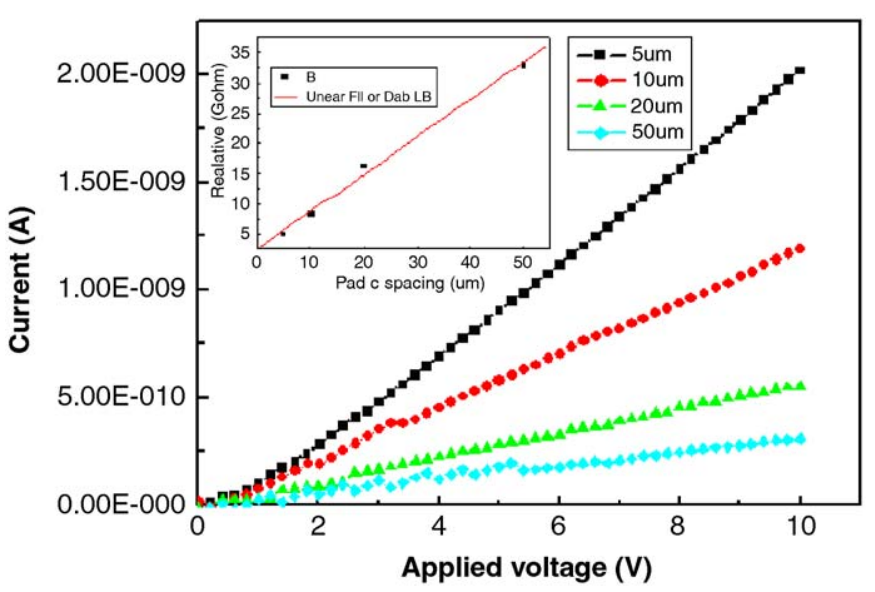

Fig. 1. $I-V$ characteristics of SI-GaN obtained from a set of TLM pads. The contact width is $200 \mu \mathrm{m}$, the spacings are 5, 10, 20 and $50 \mu \mathrm{m}$. 


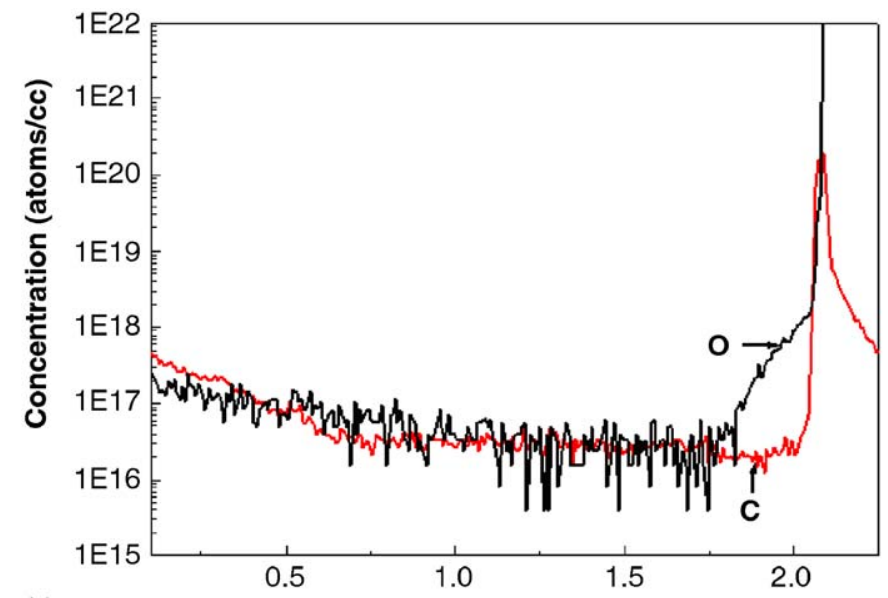

(a)

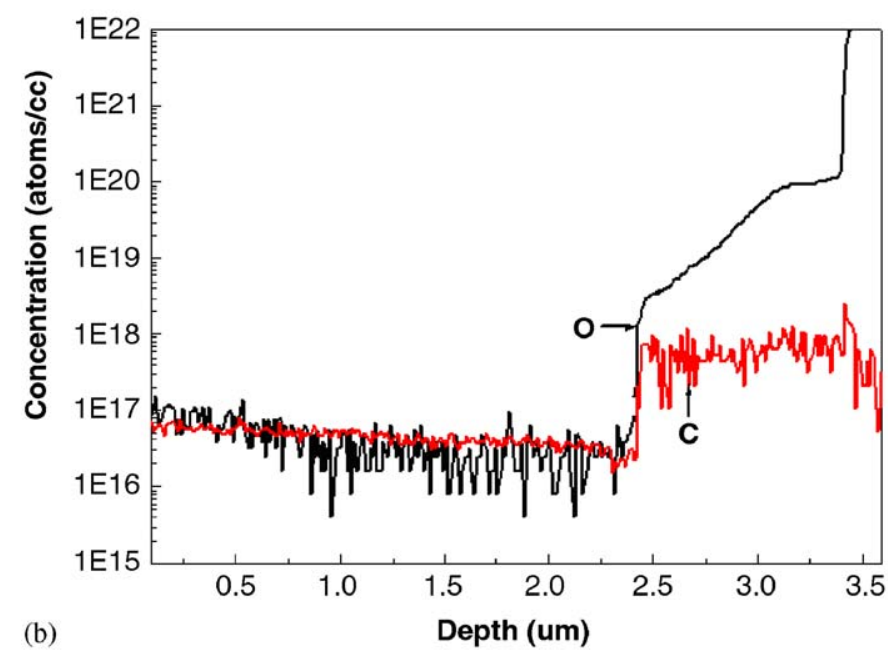

Fig. 2. SIMS profiles of $\mathrm{C}$ and $\mathrm{O}$ impurities recorded from cross-sectional normal GaN (a) and SI-GaN on AlN buffer (b).

oxygen concentration distribution in the SI-GaN is nearly the same as that in normal GaN away from the substrate (as shown in Fig. 2(a)), probably due to the constant growth parameters of the HT-GaN. It is noteworthy that the oxygen concentration shows a sharp gradient, decreasing from $5 \times 10^{18} \mathrm{~cm}^{-3}$ to less than $1 \times 10^{17} \mathrm{~cm}^{-3}$ at the interface of the $\mathrm{GaN}$ and $\mathrm{AlN}$ buffer, indicates that the oxygen atoms did not diffused from the AlN buffer into the GaN subsequent layer. The carbon impurity concentrations in the semi-insulating and normal $\mathrm{GaN}$ are also nearly the same due to the same growth parameters. It seems that the electrical property of undoped $\mathrm{GaN}$ film depends strongly on the lower buffer layer: GaN film grown on high resistance AlN buffer show semi-insulating electrical character; while grown on high conductive $\mathrm{GaN}$ channel show n-type conductive property. Therefore, we deduced that the MOCVD as-grown GaN layer is natural semi-insulating material. The top part of normal $\mathrm{GaN}$ is also high resistance and the high conductivity is from the thin degenerate layer near the sapphire interface. This can explain why the SI-GaN can be obtained with Fe doing only in the first $0.3 \mu \mathrm{m}$ of high temperature growth, and the $\mathrm{GaN}$ growth on $\mathrm{SiC}$ substrates typically gives semiinsulating character without any special technique [7].

To improve the crystal quality of the GaN layer, the DTGaN was introduced between the AlN buffer and HT$\mathrm{GaN}$, as shown in Fig. 3. The growth temperatures of the DT-GaN were altered from 1000 to $1050{ }^{\circ} \mathrm{C}$, while the growth rate and $\mathrm{V} / \mathrm{III}$ ratio were kept at $1.2 \mu \mathrm{m} \mathrm{h}^{-1}$ and 860 for all of the samples. To quantify the influence of the growth temperature on structural properties of GaN, XRD rocking scans ( $\omega$ scans) measurements were performed. The full-width at half-maximum (FWHM) of symmetric $\left(\begin{array}{ll}0 & 2\end{array}\right)$ peak in $\mathrm{GaN}$ is sensitive to the screw and mixed type threading dislocations (TDs) density in the layer, while the FWHM of asymmetric (1 02 ) peak is sensitive to all kinds of TDs [16]. Both symmetric (l 002$)$ and asymmetric (lll 02$)$ rocking curves were obtained from the samples. Fig. 4 shows the dependence of FWHM of XRD from $\left(\begin{array}{lll}0 & 0 & 2\end{array}\right)$ and (1 02 ) reflections on the DT-GaN growth temperature. It reveals that the TDs in GaN demonstrate a remarkable relation with the growth temperature of DT-GaN. The minimum FWHM values of both (0 $\left.\begin{array}{lll}0 & 2\end{array}\right)$ and $\left(\begin{array}{lll}1 & 0 & 2\end{array}\right)$ curves were obtained when the DT-GaN was grown at $1020^{\circ} \mathrm{C}$. The FWHM of the XRD rocking curve of normal GaN that is was 236 and 385 arcsec for (002) and (102) reflections, respectively. However, the FWHM of GaN grown on AlN buffer with optimized DT-GaN interlayers reveal low values of 91 and 240 arcsec for $\left(\begin{array}{lll}0 & 0 & 2\end{array}\right)$ and $\left(\begin{array}{ll}1 & 02\end{array}\right)$ reflections, respectively. When the growth temperature of DT-GaN is $1000^{\circ} \mathrm{C}$, the crystal quality of $\mathrm{GaN}$ is deteriorated due to the low growth temperature.

\section{$\mathrm{HT}-\mathrm{GaN} \sim 2.0 \mu \mathrm{m}$}

\section{Mid-temp $\mathrm{GaN} \sim 0.5 \mu \mathrm{m}$}

$$
\text { HT-AIN buffer } \sim 1.0 \mu \mathrm{m}
$$

$$
\text { LT-AIN nucleation 15nm }
$$

\section{Sapphire substrate}

Fig. 3. Schematic drawing of the epi-growth structure with the insertion of a DT-GaN layer. 


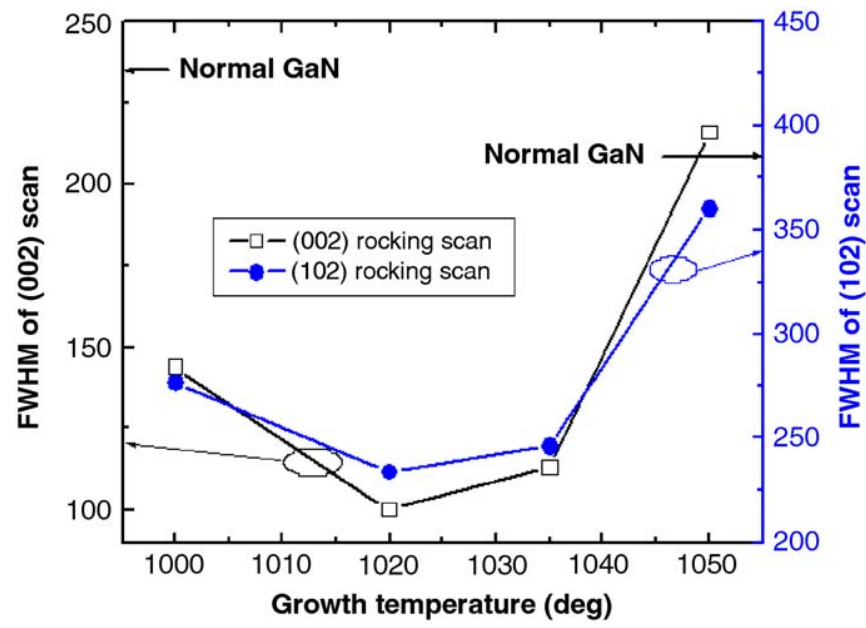

Fig. 4. Dependence of FWHM of XRD from (0002) and (1 $\left.0 \overline{1}_{2}\right)$ reflections on the DT-GaN growth temperature.

Recently, we demonstrated the highly improved structural properties of SI-GaN grown on AlN buffer/ sapphire, in comparison with the SI-GaN grown on sapphire substrate using the two-step method [13]. In situ reflectance trace showed that our $\mathrm{GaN}$ growth on high-temperature AlN buffer is initiated from a threedimensional to two-dimensional mode due to the lattice mismatch between GaN and AlN. It was reported that the $\mathrm{GaN}$ grown on AlN buffer is initiated from either a threedimensional or a two-dimensional mode, depending on the growth parameters $[17,18]$. In this research, we found that the recovery time (the time taken for the reflectance from the onset of growth to fully recovered stable oscillations) is prolonged by decreasing the growth temperature. Delaying of the coalescence enables the size of island-like seeds to increase, and allows for more of the edge-type TDs to annihilate by bending $90^{\circ}$, leading lower density of TDs in GaN layer [19].

A HEMT structure was grown based on the SI-GaN template with $25 \mathrm{~nm} \mathrm{Al}_{0.3} \mathrm{Ga}_{0.7} \mathrm{~N}$ top layer and $2 \mathrm{~nm}$ AlN barrier between $\mathrm{AlGaN}$ and $\mathrm{GaN}$. All the layers were undoped. Hall-effect measurement was performed on the HEMT sample at room temperature. The sheet charge and mobility were $1.3 \times 10^{13} \mathrm{~cm}^{-2}$ and $1700 \mathrm{~cm}^{2} \mathrm{~V}^{-1} \mathrm{~s}$, respectively. Sample HEMT devices with $L_{\mathrm{SD}} / L_{\mathrm{SG}} / W_{\mathrm{G}} / L_{\mathrm{G}}=4 /$ $1 / 250 / 2 \mu \mathrm{m}$ were fabricated from the epilayer structure. Ti/ $\mathrm{Al} / \mathrm{Ni} / \mathrm{Au}(250 / 2200 / 400 / 500 \AA)$ was used for source and drain ohmic contacts annealed at $850^{\circ} \mathrm{C}$ for $30 \mathrm{~s}$ in nitrogen ambient. Ni/Au (400/500 ̊) Schottky gates were then metalized. A reactive ion-etched mesa was used for the device isolation. Good pinch-off DC $I_{\mathrm{DS}}-V_{\mathrm{DS}}$ characteristics of the HEMT device without passivation are shown in Fig. 5. The gate voltage starts at $+1 \mathrm{~V}$ with $1 \mathrm{~V}$ steps, giving an $I_{\mathrm{DSs}}$ of $700 \mathrm{~mA} \mathrm{~mm}^{-1}$ and a maximum transconductance of about $140 \mathrm{mS} \mathrm{mm}^{-1}$. The breakdown voltage of the device is higher than $60 \mathrm{~V}$. The Hall measurement results and device $I_{\mathrm{DS}}-V_{\mathrm{DS}}$ characteristics

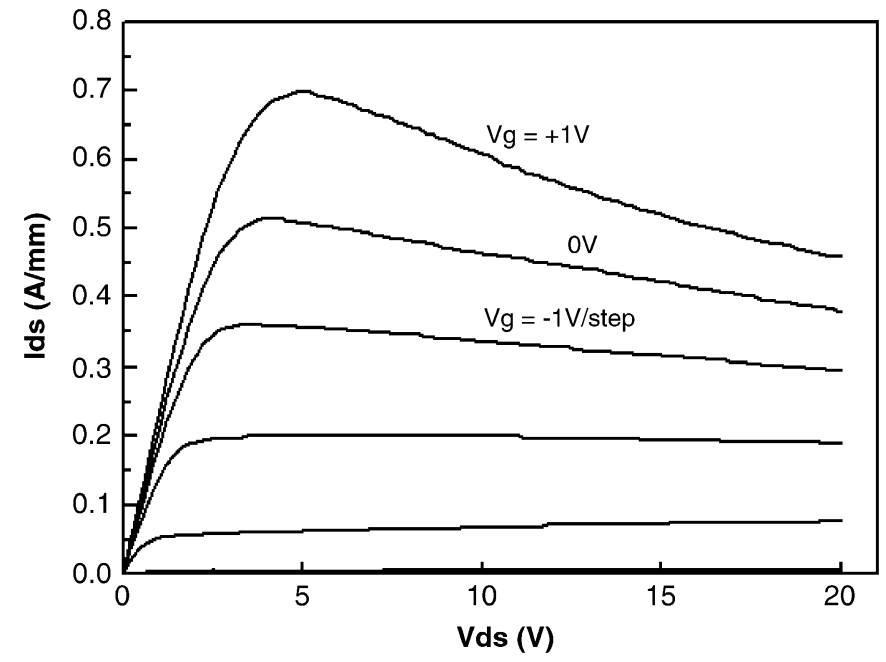

Fig. 5. $I-V$ characteristics of the HEMT sample with a $1.0 \mu \mathrm{m}$ gate based on AlN buffer/sapphire substrate.

confirmed the good crystal quality and semi-insulating character of the GaN layer on AlN buffer/sapphire substrate.

\section{Summary}

In summary, we have demonstrated the highly improved crystal quality of thick SI-GaN layers grown on an AlN buffer layer/sapphire substrate by MOCVD. It was found that the insertion of a DT-GaN interlayer between HTGaN and AlN buffer could reduce the TDs density efficiently. The crystal quality of GaN layer demonstrates remarkably dependence on the growth temperature of DT$\mathrm{GaN}$. In comparison with normal $\mathrm{GaN}$ grown on sapphire substrate, the electrical semi-insulating character of $\mathrm{GaN}$ can be achieved along with highly improved crystal quality. Moreover, we found that the MOCVD as-grown natural $\mathrm{GaN}$ is semi-insulating material and n-type conductivity of normal $\mathrm{GaN}$ is originated from the interlayer near the sapphire substrate.

\section{Acknowledgements}

This work was supported by EU-DALHM, EU NOEMETAMORPHOSE, EU NOE-PHOREMOST, TUBITAK-NANOTR, and TUBITAK under Project Nos. 104E090, 105E066. One of the authors (Ekmel Ozbay) acknowledges partial support from the Turkish Academy of Sciences.

\section{References}

[1] Y.-F. Wu, D. Kapolnet, J.P. Ibbetson, P. Parikh, B.P. Keller, U.K. Mishra, IEEE Electron. Dev. Lett. 48 (2001) 586.

[2] D.-H. Youn, V. Kumar, J.-H. Lee, R. Schwindt, W.-J. Chang, J.-Y. Hong, C.-M. Jeon, S.-B. Bae, K.-S. Lee, J.-L. Lee, J.-H. Lee, I. Adesida, Electron. Lett. 39 (2003) 566. 
[3] S.M. Hubbard, G. Zhao, D. Pavlidis, W. Sutton, E. Cho, J. Crystal Growth 284 (2005) 297.

[4] W. Seifert, R. Franzheld, E. Butter, H. Sobotta, V. Riede, Crystal Res. Technol. 18 (1983) 383.

[5] C. Wetzel, T. Suski, J.W. Ager III, E.R. Weber, E.E. Haller, S. Fischer, B.K. Meyer, R.J. Molnar, P. Perlin, Phys. Rev. Lett. 78 (1997) 3923.

[6] J.B. Webb, H. Tang, S. Rolfe, J.A. Bardwell, Appl. Phys. Lett. 75 (1999) 953.

[7] S. Heikman, S. Keller, S.P. DenBaars, U.K. Mishra, Appl. Phys. Lett. 81 (2002) 439.

[8] Z. Bougrioua, M. Azize, P. Lorenzini, M. Laügt, H. Haas, Phys. Stat. Sol. A 202 (2005) 536.

[9] A.Y. Polyakov, N.B. Smirnov, A.V. Govorkov, S.J. Pearton, J. Vac. Sci. Technol. B 22 (2004) 120

[10] Z. Bougrioua, I. Moerman, L. Nistor, B. Van Daele, E. Monroy, T. Palacios, F. Calle, M. Leroux, Phys. Stat. Sol. A 195 (2003) 93.
[11] S. Arulkumaran, T. Egawa, H. Ishikawa, T. Jimbo, Appl. Phys. Lett. 81 (2002) 3073.

[12] B. Heying, E.J. Tarsa, C.R. Elsass, P. Fini, S.P. DenBaars, J.S. Speck, J. Appl. Phys. 90 (2001) 5196.

[13] H.B. Yu, D. Caliskan, E. Ozbay, J. Appl. Phys., accepted for publication.

[14] D.C. Look, R.J. Molnar, Appl. Phys. Lett. 70 (1997) 3377.

[15] M.G. Cheong, K.S. Kim, C.S. Oh, N.W. Namgung, G.M. Yang, C.-H. Hong, K.Y. Lim, E.-K. Suh, K.S. Nahm, H.J. Lee, D.H. Lim, A. Yoshikawa, Appl. Phys. Lett. 77 (2000) 2557.

[16] B. Heying, X.H. Wu, S. Keller, Y. Li, D. Kapolnek, B.P. Keller, S.P. DenBaas, J.S. Speck, Appl. Phys. Lett. 68 (1996) 643.

[17] Y. Ohba, S. Iida, Jpn. J. Appl. Phys. 41 (2002) L615.

[18] H. Jiang, T. Egawa, M. Hao, Y. Liu, Appl. Phys. Lett. 87 (2005) 241911.

[19] J. Chen, S.M. Zhang, B.S. Zhang, J.J. Zhu, G. Feng, X.M. Shen, Y.T. Wang, H. Yang, W.C. Zheng, J. Crystal Growth 254 (2003) 348. 